\title{
A STATISTICAL SUBSPACE METHOD FOR BLIND CHANNEL IDENTIFICATION IN OFDM COMMUNICATIONS
}

\author{
Xiangyang Zhuang ${ }^{1}$, Zhi Ding ${ }^{2}$ and A. Lee Swindlehurst ${ }^{1}$ \\ ${ }^{1}$ Dept. of Elec. \& Comp. Engineering, Brigham Young University, Provo, UT 84602 \\ ${ }^{2}$ Dept. of Elec. \& Comp. Engineering, University of Iowa, Iowa City, IA 52242 \\ email:jeffz@ee.byu.edu,zding@eng.uiowa.edu, swindle@ee.byu.edu
}

\begin{abstract}
This paper presents a subspace method for blind channel estimation based on a special output correlation matrix. The approach relies on the i.i.d. assumption of the data sequence and uses the cyclic prefix redundancy present in OFDM systems or single-carrier systems with frequency domain equalization. This method has an important feature that allows channels to be longer than the cyclic prefix. In addition, unknown frame synchronization can be accommodated. There is no constraint on the zero locations of the channel and the performance is asymptotically independent of white noise. The method is compared with a simple correlation approach and a deterministic subspace method.
\end{abstract}

\section{INTRODUCTION}

For high speed communications, OFDM (Orthogonal Frequency Division Multiplexing) has recently emerged as a viable scheme that allows a simple channel equalization through frequency domain multiplication rather than timedomain deconvolution. Channel estimation can even be avoided if differential PSK modulation is used [6]. However, differential demodulation suffers a typical $3 \mathrm{~dB}$ SNR loss compared with coherent demodulation which requires channel knowledge. To estimate the channel, both training and blind identification methods can be applied. In broadcasting or multicasting systems, periodic training data frames can significantly reduce the overall system channel capacity. Thus, blind channel identification becomes an attractive alternative.

In this paper, we present a blind channel estimation algorithm which exploits only the transmission redundancy induced by the cyclic prefix (CP) in an OFDM system. The method also applies to single-carrier (SC) systems that also use CP for frequency domain equalization. The pros and cons of OFDM and SC communications in terms of carrier offset sensitivity, peak-to-average ratio and other factors can be found in [1][6]. Our method exploits secondorder statistics of the received signal under the assumption that the source sequences is independent and identically distributed (i.i.d.). Channels longer than the CP can also be identified, which is a merit useful for dispersive channels under-accommodated by the $\mathrm{CP}$ guard interval. OFDM symbol synchronization requirement can be relaxed by over-estimating the channel response padded with zeros at both ends. The additional zeros in the channel response have little effect on the algorithm and the estimation performance is asymptotically independent of the white noise. We will compare our method with two statistical approaches reported in [4] and a deterministic (without the i.i.d. assumption) subspace method in [5], all of which assume that symbol synchronization is achieved and that the unknown channel response is shorter than the CP.

\section{SYSTEM MODEL AND PROBLEM FORMULATION}

In an OFDM transmitter, channel-encoded serial data stream is first segmented into length- $N$ blocks often referred to as OFDM symbols. We denote the $k^{\text {th }}$ OFDM symbol by $\mathrm{s}(k)=\left[s_{1}(k), s_{2}(k), \cdots, s_{N}(k)\right]^{T}$ on which IDFT is applied The last $D$ samples of the $N$-IDFT outputs are repeated in front of the $N$-IDFT outputs as the so-called cyclic prefix (CP). This forms a $(N+D)$ sample frame which will be transmitted in serial. The $N$-point IDFT can be described as $F \cdot \mathbf{s}(k)$ where $F$ is a $N$-by- $N$ IDFT matrix. Denoting $F_{D}$ as the sub-matrix consisting of the last $D$ rows of the $F$, we can write the transmitted $k^{\text {th }}$ frame as $\mathbf{x}(k)=\left[F_{D}^{T}, F^{T}\right]^{T} \mathbf{s}(k)$. In an SC transmitter, however, no IDFT is performed and the CP is just the repetition of the last $D$ data symbols.

The received baseband signal is sampled at the baud rate. The sampled data are segmented into frames of size$(N+D)$ with (or without) the knowledge of the transmitted frame boundary. The dispersive channel is of order $L_{h}$, which is denoted as $\mathbf{h}=\left[h_{0}, h_{1}, \cdots, h_{L_{h}}\right]^{T}$. We assume, for notational simplicity, that the OFDM symbol size $N$ is an integer multiplication of the length of CP (i.e., $N=M D$ ), and we would like to represent the received and transmitted frames in sub-blocks of size- $D$ whose boundaries are aligned with the CP block. Since we will use correlation between adjacent frames, we write two successive received frames as:

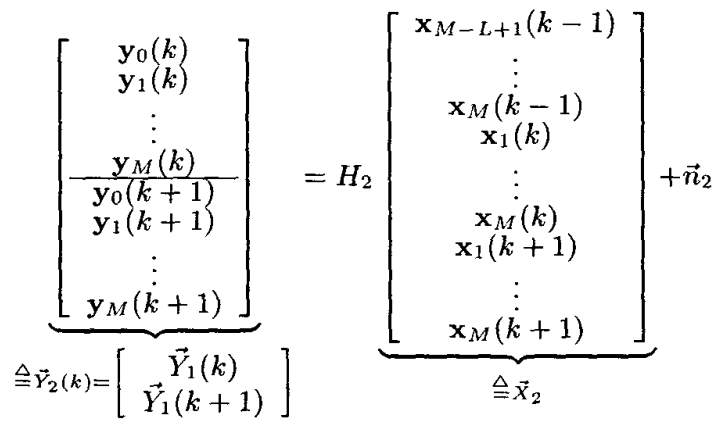

where $\mathbf{x}_{i}(k)\left(\mathbf{y}_{i}(k)\right)$ denotes the $i^{\text {th }}$ size- $D$ sub-block of the 
$k^{\text {th }}$ transmitted (received) frame, $\vec{n}_{2}$ is the noise, and

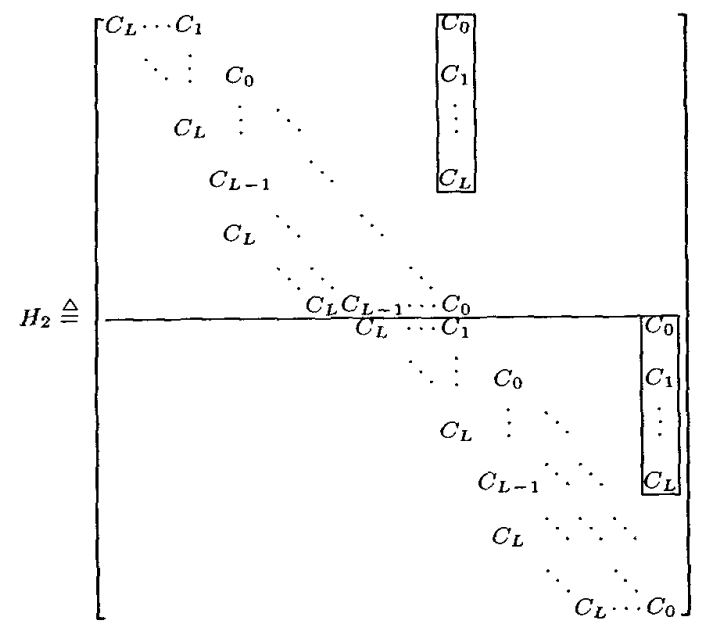

$C_{0}, C_{1}, \cdots, C_{L}$ are $D$-by- $D$ matrices partitioning a Toeplitz matrix formed from h, i.e., (assuming known frame boundary)

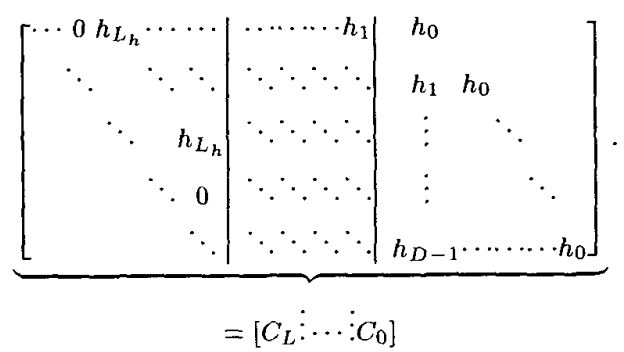

In (2), the boxed block is in that position because the CP block equals to the last sub-block of each frame (That is why we want to align sub-blocks with the CP). The boxed column will not overlap with the $C_{0}$ block below if $L \leq$ $M-1$. This bound on $L$ also limit the maximum channel order allowed by our method to $(M-1) D$. The above data model allows a description of all the methods to be discussed later. Note from (3), $C_{0}$ is a lower triangular matrix with diagonal entry $h_{0}$ and $C_{L}$ is upper triangular with possibly zero diagonal (or $h_{L_{h}}$ ). To cover the whole channel, $L=\left\lceil\frac{L_{h}}{D}\right\rceil\left(\lceil\cdot\rceil\right.$ is the ceiling function) such $C_{i}$ blocks are needed.

In the case that the frame boundary has an unknown lag of $p D-q$ where, without loss of generality, we can always choose $D>q>0$ and vary $p$. If $p=0, q \neq 0$ (i.e., ahead of the true boundary by $q<D$ ), we can accommodate this $q$ factor by over-estimating the channel from both ends as explained below. The sub-block boundaries are kept in line with the CP block so that we can still use the data model of ( 1 . But now, the partition lines in (3) will move to the right by $q$ columns, i.e., in the new $C_{0}, h_{0}$ retreats to the $(-q)^{t h}$ diagonal (i.e., below the main diagonal by $q$ ). However, we still estimate $C_{0}$ as a "full" lower triangular matrix and $C_{L}$ as "full" upper triangular. Therefore, the channel order is over-estimated as $L D$. In order to cover the whole channel, the number of $C_{i}$ blocks needs to be $L=\left\lceil\frac{L_{h}+q}{D}\right\rceil \leq\left\lceil\frac{L_{h}-1}{D}\right\rceil+1$. By setting $L=\left\lceil\frac{L_{h}-1}{D}\right\rceil+1$, we can guarantee the accommodation of any unknown $q$.
When the frame boundary is off by more than one subblock (e.g., if $p>0$ ), we denote one received frame as $\tilde{Y}_{1}$. Now, $\tilde{Y}_{1}$ corresponds to rows $p$ to $(p+M)$ of $\vec{Y}_{2}$, i.e., (in Matlab notation)

$$
\tilde{Y}_{1}=\vec{Y}_{2}[p D+1:(p+M) D] .
$$

Thus, $\tilde{Y}_{1}$ corresponds to matrix $\mathcal{H}_{2}[p D+1:(p+M) D,:]$.

3. ALGORITHMS BASED ON CORRELATIONS

IDFT and DFT satisfy the perfect reconstruction property, i.e., $F F^{H}=I$ where $F^{H}=F^{-1}$ is the DFT matrix. For single-carrier systems, we simply have $F=I_{N \times N}$. Under the assumption of i.i.d. source, we can verify that

$$
E\left\{\vec{X}_{2} \vec{X}_{2}^{H}\right\}=I
$$

\subsection{Existing Approaches for Shorter Channels}

A very simple correlation method is proposed in [4] where the frame boundary is assumed known and the channel is shorter than the CP, i.e., $L_{h}<D$. Only two matrices $C_{0}$ and $C_{1}$ exist in this case. Observing from (1) and (2), we have the following correlation which holds asymptotically between the first and last sub-block of the same received frame (assuming white noise):

$$
E\left[y_{0}(k) y_{M}^{H}(k)\right]=C_{0} C_{0}^{H}
$$

Because $C_{0}$ is lower triangular, the first column of $C_{0} C_{0}^{H}$ is $h_{0}^{*}\left(h_{0}, \cdots, h_{D-1}\right)$. Thus, the whole channel can be estimated up to a complex factor of $h_{0}^{*}$ if $L_{h}<D$ and $h_{0} \neq 0$. This approach, while computationally very simple, has a performance heavily depending on $h_{0}$. If we simply ignore the preceding small coefficients and designate $h_{0}$ as the first "large" channel coefficient, we actually use some other column of $C_{0} C_{0}^{H}$ which is not of the form $h_{0}^{*}\left(h_{0}, \cdots, h_{D-1}\right)$. The approximation error may not be negligible.

In the same paper[4], another approach is proposed where $C_{0}$ is estimated from the Cholesky decomposition of $C_{0} C_{0}^{H}$. Instead of one column of $C_{0} C_{0}^{H}$, more information is used. The performance still depends on a good conditioning of matrix $C_{0} C_{0}^{H}$ which requires a large $h_{0}$ as well. In implementation, since the empirical expectation $E\left[y_{0}(k) y_{M}^{H}(k)\right]$ is often not positive definite as it only approximates $C_{0} C_{0}^{H}$ asymptotically, some remedies have to be implemented to enforce its positive-definiteness.

\subsection{Channels with Longer Impulse Responses}

If the channel is longer than the CP (i.e., $L_{h} \geq D$ ), $C_{0}$ contains only part of the channel $\left(h_{0}, \cdots, h_{D-1}\right)$. In this case, not only correlations between $\mathrm{y}_{0}(k)$ and $\mathrm{y}_{M}(k)$ of the same received frame, but also between $y_{0}(k)$ and sub-blocks in the next frames must be used. Assuming known $L$, we can observe from (1) and (2) that

$$
\begin{gathered}
E\left\{\mathbf{y}_{0}(k)\left[\mathbf{y}_{M}^{H}(k), \mathbf{y}_{0}^{H}(k+1), \cdots, \mathbf{y}_{L-1}^{H}(k+1)\right]\right. \\
=\left[C_{0} C_{0}^{H}, C_{0} C_{1}^{H}, \cdots, C_{0} C_{L}^{H}\right]
\end{gathered}
$$

Using the lower triangular structure of $C_{0}$, we can now estimate the entire channel from the first row of the above correlation matrix which is $h_{0}\left(h_{0}^{*}, \cdots, h_{L D-1}^{*}\right)$. The Cholesky decomposition approach can not be used here since only $C_{0}$ is lower triangular. When frame boundary is unknown such as the $q \neq 0, p=0$ case, we need to detect $q$ since now the $(q+1)^{\text {th }}$ row contains the channel. 


\subsection{A New Algorithm Based on Correlations}

To alleviate the channel estimation sensitivity to $h_{0}$, we exploit more correlation information between sub-blocks involving two received frames. From (1) and (2), we can observe that

$$
E\left\{\vec{Y}_{1}(k)\left[\begin{array}{c}
\mathbf{y}_{M}(k) \\
\vec{Y}_{1}(k+1)
\end{array}\right]^{H}\right\}=\underbrace{\left[\begin{array}{cccccc}
C_{0} C_{0}^{H} & C_{0} C_{1}^{H} & \cdots & C_{0} C_{L}^{H} & \mathbf{0} & \cdots \\
* & C_{1} C_{1}^{H} & \cdots & C_{1} C_{L}^{H} & \mathbf{0} & \cdots \\
\vdots & \vdots & \ddots & \vdots & \mathbf{0} & \cdots \\
* & * & \cdots & C_{L} C_{L}^{H} & \mathbf{0} & \cdots \\
\mathbf{0} & \mathbf{0} & \cdots & \mathbf{0} & \mathbf{0} \\
\vdots & \vdots & \vdots & \vdots & \vdots
\end{array}\right]}_{\triangleq R}
$$

where "*" denotes correlation blocks whose exact expressions depend on the specific value of $L$ and $M$. They can have the form of $C_{i} C_{j}^{H}$, for example, when $L=1$. In this case, we might also want to use them. But we shall not pursue this tangent for now. Instead, We shall only exploit the the upper triangular part of $R$. As in the simple correlation method, the correlations of white noise is asymptotically zero since they are between blocks that are at least $M$ blocks apart. We extract the upper triangular part of $R$ and add to its Hermitian to form a matrix as an outer-product of the form $\mathcal{C C}^{H}$ where

$$
\mathcal{C}=\left\{C_{0}^{T}, C_{1}^{T}, \cdots, C_{L}^{T}, \mathbf{0}, \cdots\right]^{T}
$$

Note that $\mathcal{C}$ is Toeplitz and it is always of full column rank$D$ no matter how many preceding (or trailing) zeros are at both ends of the channel. Now a small $h_{0}$ will not cause any problem and the unknown boundary $q$ does not need to be detected because we over-estimate the channel at both ends. In addition, the zero location of the channel will not cause ill-conditioning of $\mathcal{C}$ either. In implementation, we will not compute and include those zero blocks in $\mathcal{C}$ if we know an upper bound of $L$ since too many parameter constraints will degrade the estimation accuracy.

Denote $R_{L}$ as the $(L+1) D$-by- $(L+1) D$ Hermitian matrix formed from $R$ that asymptotically equals to the non-zero part of $\mathcal{C} C^{H}$. From the Toeplitz structure of $\mathcal{C}$, a subspace method can then be adopted as in [3][2]. From the null space of $R_{L}$ which consists of $L D$ vectors of length $(L+1) D$, a Hankel matrix $G_{i},(i=1 \cdots L D)$ of size $L D$-by- $(D+1)$ is formed for each null vector. The eigenvector corresponding to the smallest eigenvalue of $\sum_{i=1}^{L D} G_{i} G_{i}^{H}$ is an estimation of the channel up to a complex scalar. We may be able to use a minimum (and possibly more reliable) noise subspace instead of all $L D$ null vectors to estimate the channel.

When the frame boundary is in error by more than one sub-block (i.e., $p>0$ ), we will label received data as successive sub-blocks $\tilde{\mathbf{y}}_{i}$ without assigning any frame index. We observe the following correlations between proper sub-blocks in $\tilde{Y}_{1}=\left[\tilde{\mathbf{y}}_{0}^{T}, \cdots, \tilde{\mathbf{y}}_{M}^{T}\right]^{T}$ and $\tilde{Y}_{2}=$ $\left[\tilde{\mathbf{y}}_{M}^{T}, \cdots, \tilde{\mathbf{y}}_{2 M}^{T}, \tilde{\mathbf{y}}_{2 M+1}^{T}, \cdots, \tilde{\mathbf{y}}_{3 M+1}^{T}\right]^{T}$, i.e.,

$$
E\left\{\tilde{Y}_{1} \tilde{Y}_{2}^{H}\right\}=\tilde{R}
$$

where (assuming $p<L$ in the representation below)

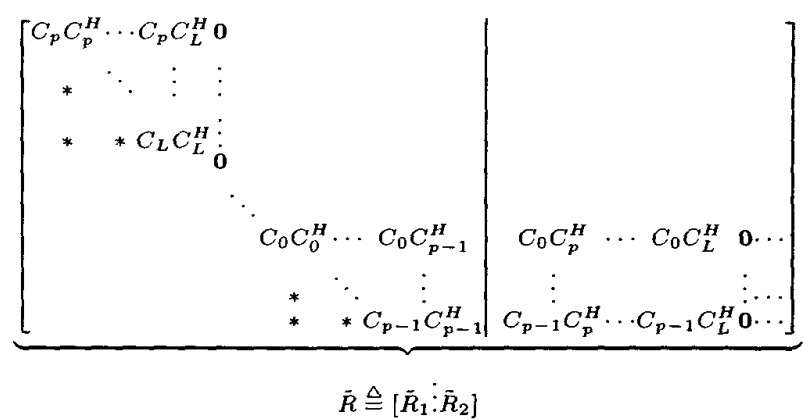

We see that the upper triangular part of $\tilde{R}_{1}$ resembles that of an outer-product structure of a circularly shifted version of $\mathcal{C}$, which is,

$$
\tilde{\mathcal{C}}=\left[C_{p}^{T}, \cdots, C_{L}^{T}, \mathbf{0}, \cdots, \mathbf{0}, C_{0}^{T}, \cdots, C_{p-1}^{T}\right]^{T}
$$

except that the $(L-p+1) D$-by- $p D$ upper right corner block of $\tilde{R}$ consists of asymptotic zeros instead of what is should be in $\tilde{\mathcal{C}} \tilde{C}^{H}$. In order to obtain the desired outer-product, we can replace that corner with the Hermitian of the non-zero part of $\bar{R}_{2}$ after we detect $p$ or equivalently, the location of $C_{0}$. There are structured information in $\tilde{R}_{1}$ that can be useful in detecting $p$. For example, there usually exist consecutive zeros between $h_{L_{h}} h_{L_{h}}^{*}$ (last non-zero diagonal entry of the $C_{L} C_{L}^{H}$ block) and $h_{0} h_{0}^{*}$ (first non-zero diagonal entry of the $C_{0} C_{0}^{H}$ block). Additionally, only $C_{0} C_{0}^{H}$ has all the correlation blocks directly above it being zeros.

We even can choose not to estimate $p$ by estimating the channel at its maximum allowed order $L_{h}=$ $(M-1) D$. More specifically, by adding the Hermitian of $\left.E\left\{\tilde{\mathbf{y}}_{i-1}\left[\tilde{\mathbf{y}}_{2 M}^{H} \cdots \tilde{\mathbf{y}}_{2 M+i-2}^{H}\right]\right\}\right)$ back to $\tilde{R}_{1}[1:(i-1) D,(i-$ 1) $D+1: i D]$ for all $i=2, \cdots, M+1$, we will get the desired outer-product of $\tilde{\mathcal{C}} \tilde{\mathcal{C}}^{H}$ because either the added or the original correlation block (or both) is asymptotically a zero block. Thus, we can estimate a circular shifted channel with order $L_{h}=(M-1) D$ to avoid any attempt to estimate the unknown boundary factor. However, if too many coefficients are estimated, the estimation accuracy will be degraded. Moreover, the $h_{0}$ position may still be needed to finalize the channel identification since a circularly shifted channel is not good enough for equalization.

\subsection{Comparison with a Deterministic Method}

While our method is based on i.i.d, assumption, another subspace method was proposed where the i.i.d. assumption is relaxed to requiring "sufficient" number of OFDM frames [5] for persistent excitation. The algorithm was originally for the synchronized case with $L_{h}<D$ (i.e., $L=1$ ), but it can be extended to the $L_{h}>D$ case as follows. Note from (1) that one frame corresponds to a $(M+1) D$-by$(M+L) D$ matrix (the upper half of $\left.H_{2}\right)$. By stacking at least $L+1$ consecutively received frame, a large matrix $\mathcal{H}$ of size $(L+1)(M+1) D$-by- $(L+M+L M) D$ is formed which has more rows than columns and will be full column rank. As long as the source autocorrelation matrix is of full column rank which requires the collection of at least $(L+M+L M) D$ frames, the null subspace of the correlation 
matrix can be employed to estimate the channel. Now, a correlation matrix of size $(L+1)(M+1) D$-by- $(L+M+$ $L M) D$ as opposed to size $(L+1) D$-by- $(L+1) D$ (i.e, $R_{L}$ in our method) is computed. The eigen-decomposition is more complex too. The performance of this deterministic subspace method is not affected by the numerical value of $h_{0}$ either while $q \neq 0$ is also allowed. However if $p>0$, one frame will correspond to $\mathcal{H}_{2}[p D+1:(p+M) D,:]$. It can be shown that more received frames (at least $M+L$, instead of $L+1$ ) have to be stacked in order to result in a "tall" channel matrix. Even if the prohibitively high computation burden can be tolerated, $p$ still remains known because the structure of that channel matrix must be known in order to use the subspace approach.

\section{SIMULATIONS}

We consider the case of direct OFDM broadcasting system. The wireless channel we use is generated according to the "Typical Urban" profile specified by the European COST207 project, the symbol rate is set to be $4 \mathrm{M}$ Baud/s and a stationary channel is assumed (i.e., no Doppler shift) The modulation is 16-QAM. Channel vector is normalized to norm 1 , so the input SNR is $10 \log \left(\sigma_{s}^{2} / \sigma_{n}^{2}\right)$. Our measurement is the channel mean-squared estimation error $M S E \triangleq\|\hat{\mathbf{h}}-\mathbf{h}\|^{2}$ averaged over 100 Monte Carlo runs.

We first consider the synchronized case (i.e., $p=q=0$ ) with $D=8$ and $M=4$. To compare our method to those in [4] and [5], we truncated channels to $L_{h}=7$ such that $h_{0}$ is the second biggest coefficient. Even for this $h_{0}$, our method always out-performs the simple correlation. It also out-performed the deterministic method under low SNR or small number of frames.
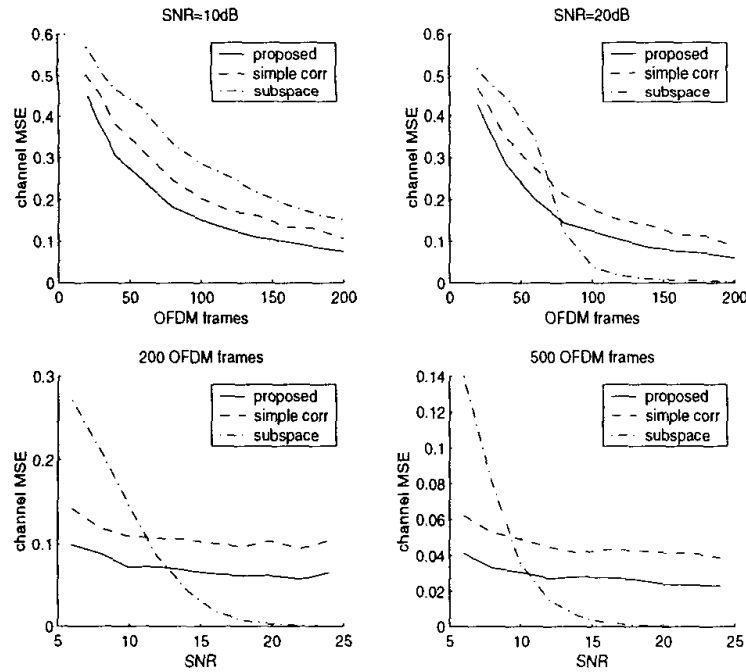

Figure 1: Synchronized Case with Large $h_{0}$ and $L_{h}<D$

In Figure 2, we include one more preceding channel coefficient which is still about $1 / 8$ of the peak value. Not surprisingly, the simple correlation method provides errant results.

Next in Figure 3, we consider the case where $D=4$ but $L_{h}=12$, i.e., the channel is longer than the CP. We also
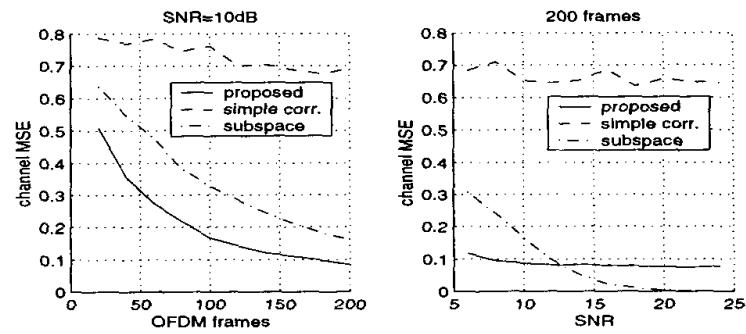

Figure 2: Synchronized Case with Small $h_{0}$ and $L_{h}=D$ include an unknown lag of $q=3$. To compare with the deterministic subspace method, we assume $p=0$. Due to the unknown $q, 5$ sub-blocks are needed to cover the channel (i.e., $L=4$ ). Here, we actually estimated a channel of order $L D=16$.
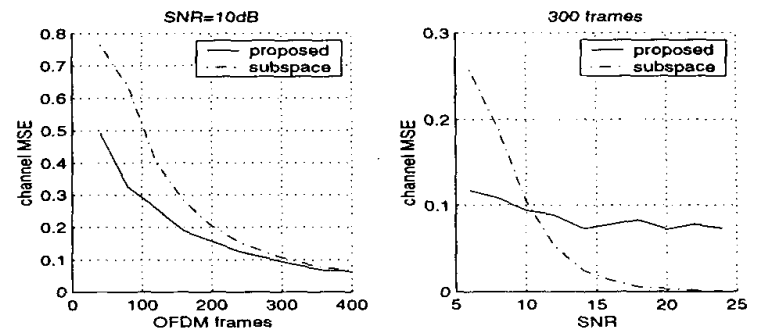

Figure 3: Unknown Boundary Case with $L_{h}>D$

\section{REMARKS}

Due to the i.i.d. assumption, our subspace method still require many frames to obtain good statistical information, while the deterministic subspace method also needs sufficient frames. Hence, they may be applicable to only stationary multicasting systems. The CP can be viewed as a special block code, provides an alternative for blind channel estimation in a single-carrier system if a multichannel setting (e.g., oversampling or antenna array) does not exist. This is akin to transmitter-induced cyclostationarity proposed in [7]. In fact, the repetition coding and interleaving scheme in [7] correspond to the $L_{h}<D$ and $M=1$ case.

\section{REFERENCES}

[1] A. Czylwik. Comparison between adaptive ofdm and single carrier modulation with frequency domain equalization. Proc. IEEE Vehicular Technol. Conf., pages 865-869, 1997.

[2] Z. Ding. A cumulant matrix subspace algorithm for blind single fir channel identification. Proc. IEEE Sig. Pro. Workshop on H.O.S., June 1999

[3] E. Moulines, P. Duhamel, J.-F. Cardoso, and S. Mayrargue. Subspace methods for the blind identification of multichannel FIR filters. IEEE Trans. Signal Process., 43:516-525, Feb. 1995.

[4] B. Muquet and M. de Courville. Blind and semi-blind channel indentification methods using second order statistics for OFDM systems. IEEE ICASSP Proc., Mar. 1999.

[5] B. Muquet, M. de Courville, P. Duhamel, and V. Buzenac. A subspace based blind and semi-blind channel indentification methods for OFDM systems. IEEE SPAWC Proc., June 1999.

[6] H. Sari, G. Karam, and I. Jeanclaude. Transmission techniques for digital terrestrial TV boradeasting. IEEE Commun. Mag., pages 100-109, Feb. 1995.

[7] M. K. Tsatsanis and G. B. Giannakis. Transmitter induced cyclostationarity for blind channel equalization. IEEE Trans. Sig. Process., pages 1785-1794, July 1997. 